CORRECTION

https://doi.org/10.1038/s41586-018-0116-0

\title{
Author Correction: Roads towards fault-tolerant universal quantum computation
}

Earl T. Campbell, Barbara M. Terhal \& Christophe Vuillot

Correction to: Nature https://doi.org/10.1038/nature23460, published online 13 September 2017.

In Fig. 2b of this Review, two of the gates were inadvertently swapped. At the top right, ' $X^{a+c}$ ' should have been ' $Z$ ', and at the bottom right ' $Z$ ' ' should have been ' $X^{a+c}$ '. Fig. $2 \mathrm{~b}$ has been corrected online. The Supplementary Information of this Author Correction contains the original, incorrect Fig. 2b, for transparency.

Supplementary Information is available in the online version of this Amendment. 